\section{Cómo implementar la nueva ley chilena antitabaco al interior de la universidad}

\author{
Baltica Cabieses, ${ }^{1}$ Mónica Muñoz, ${ }^{2}$ \\ Soledad Zuzulich y Aixa Contreras ${ }^{1}$
}

Forma de citar: Cabieses B, Muñoz M, Zuzulich S, Contreras A. Cómo implementar la nueva ley chilena antitabaco al interior de la universidad. Rev Panam Salud Publica. 2008;23(5):361-8.

Palabras clave: servicios de salud para estudiantes, promoción de la salud, campañas para el control del tabaquismo, Chile.

\footnotetext{
1 EM, Miembro Programa UC Saludable. Pontificia Universidad Católica de Chile. La correspondencia deberá dirigirse a Baltica Cabieses, Vicuña Mackenna 4860, Macul Santiago 6904411, Chile. T.: 00562 3545303 F.: 00562354 7025. Correo electrónico: bcabiese@uc.cl

2 Directora, Programa UC Saludable. Pontificia Universidad Católica de Chile. Escuela de Enfermería.
}

En el Diario Oficial de la República de Chile se publicó, el 16 de mayo de 2006, la Ley $N^{\circ} 20.105$, que modificó a la $\mathrm{N}^{\mathrm{o}} 19.419$ concerniente a la publicidad y el consumo de tabaco (1). Desde que esta ley entró en vigor el 14 de agosto del mismo año, diversas instituciones y espacios públicos han debido acatar sus disposiciones. Con respecto a las instituciones de educación superior, dicha legislación ha determinado claramente sus exigencias, así como las consecuencias de su incumplimiento, lo cual ha motivado a las universidades a buscar estrategias de implementación eficientes, oportunas y duraderas.

En el año 2000, la Pontificia Universidad Católica de Chile (UC) manifestó la necesidad de complementar su proyecto con un programa que propiciara el incremento de la calidad de vida de la comunidad universitaria. Surgió así el Programa UC Saludable, cuya meta es incorporar —en el marco de la promoción de la salud-, estrategias de intervención que propicien el desarrollo humano y mejoren la calidad de vida de quienes allí estudian o trabajan (2).

La implementación de esta ley se consideró como una línea de trabajo necesaria y relevante en la promoción de la salud al interior de la UC pues, además de responder a la contingencia de la legislación chilena, se buscaba cumplir con los tres objetivos centrales del Programa UC Saludable: (a) integrar el concepto de promoción de la salud en la cultura de la UC, sus políticas, estructura, procesos y currículo; (b) crear un entorno universitario que fomente en estudiantes, profesores y personal administrativo una vida saludable, y (c) trabajar en redes con organizaciones de educación superior y otras, interesadas en asumir la promoción de la salud como su responsabilidad social (2).

Uno de los mayores desafíos en la promoción de la salud en una institución de educación superior y en la comunidad en general, es su incorporación sostenida como elemento relevante y pertinente de considerar en las diversas estrategias e innovaciones organizacionales. Si bien la promoción puede ser considerada como un eje transversal en el mejoramiento de la calidad de vida de la población, no siempre se incluye en las discusiones entre expertos o en la toma de decisiones institucionales.

El propósito del presente artículo es dar a conocer la experiencia relativa a la implementación de la nueva ley chilena antitabaco al interior de la UC durante 2006. Se espera motivar y contribuir a la reflexión y toma de decisiones de otras instituciones 
de educación superior en este tema, y fortalecer la promoción de la salud en el entorno universitario. Así, se podría dar cabida a la promoción de la salud a través de diversas innovaciones organizacionales al interior de las universidades.

\section{LEGISLACIÓN ANTITABACO Y PROMOCIÓN DE LA SALUD}

La experiencia que se describe a continuación está focalizada en dos temas independientes y a la vez convergentes: la promoción de la salud y la nueva ley chilena antitabaco. Como lo señaló la Organización Panamericana de la Salud el año 2002, el concepto de promoción de la salud es, en sí, un proceso y no una solución fácil. Su propósito es lograr un resultado concreto en un largo plazo, pero con efectos de mediano y corto plazo que se traducen en el mejoramiento de la calidad de vida de la comunidad (3).

Si una institución de educación superior asume el desafío de ser promotora de la salud, debe entonces conocer, comprender, apoyar y fortalecer estilos de vida saludables para cada uno de los miembros de esa comunidad. Esto coincide con el concepto central de promoción de la salud que se presenta en la Carta de Ottawa de 1986: "la salud es construida y vivida por las personas en su ambiente cotidiano, donde ellas trabajan, aprenden, juegan y aman" (4). Al mismo tiempo, la universidad debe promover y apoyar la colaboración entre los diversos sectores del gobierno y los organismos no gubernamentales (5).

La nueva ley chilena antitabaco tiene como propósito promover la salud de la población a través de disposiciones que contemplan separar ambientes para fumadores y no fumadores (1), y no promover el consumo mediante la publicidad. ${ }^{3} \mathrm{De}$ acuerdo con lo descrito por diversos autores $(6,7)$ existe sustento teórico para avalar su relevancia y efectividad, puesto que las medidas actuales de control del tabaco, en el plano internacional, incluyen no sólo estrategias para desalentar el inicio del consumo y fomentar el abandono del hábito tabáquico, sino que también protegen a los no fumadores de la exposición pasiva.

Destacan de esta nueva ley las disposiciones (8) que prohíben: (a) la publicidad del tabaco salvo al interior de los lugares de venta; (b) la comercialización, ofrecimiento, distribución o entrega de tabaco a menores de 18 años, y (c) fumar en establecimientos educativos y de salud. Asimismo, se

3 Pontificia Universidad Católica de Chile. Normas para el cumplimiento de la ley Antitabaco $N^{\circ} 20.105$. Dirección de Asuntos del Personal. 2006. Santiago, Chile. [Documento de uso interno, no publicado]. establece que: (a) los espacios diseñados para fumadores deben estar claramente especificados y contar con ventilación al aire libre o artificial; (b) todo producto de tabaco debe contener información acerca de los daños y padecimientos que ocasiona, y (c) debe impartirse educación en etapa escolar básica y media sobre los efectos perjudiciales del consumo de tabaco en la salud.

\section{PROCESO DE IMPLEMENTACIÓN DE LA NUEVA LEY CHILENA ANTITABACO EN LA UNIVERSIDAD CATÓLICA}

Para describir este proceso, es necesario abordar el trabajo previo desarrollado en torno a ambientes libres de humo de tabaco (ALHT) al interior de la UC.

En una primera fase del Programa UC Saludable, se llevó a cabo un estudio diagnóstico de consumo de tabaco y otros estilos de vida en los estudiantes de la UC. Esta investigación se realizó en 2001 y consideró una muestra aleatoria de 719 alumnos, pertenecientes a 29 carreras de la UC. De los encuestados, 55\% indicaron haber fumado alguna vez en la vida, y de ellos 37\% eran fumadores activos al momento de contestar el cuestionario, con un promedio de consumo de seis cigarrillos diarios; $82 \%$ de estos últimos estaban de acuerdo en la creación de ALHT en la UC. ${ }^{4}$

A partir de los resultados del estudio diagnóstico, el Programa UC Saludable inició el proceso de certificación ministerial de la Escuela de Enfermería de la UC como ALHT, misma que se obtuvo en 2005, tras cerca de 12 meses de trabajo para cumplir con los requisitos del Ministerio de Salud de Chile, que consistieron en: a) constituir un comité representativo; b) proporcionar información y hacer difusión entre todos los involucrados acerca de la generación de ALHT; c) establecer la prohibición parcial temporal (un par de meses de marcha blanca en la Escuela de Enfermería de la UC), y después la prohibición total de fumar; d) brindar apoyo a los interesados en dejar de fumar (derivación a Dirección General Estudiantil, Área Salud, para orientación y referencia a centros expertos), y e) definir estrategias de evaluación del cumplimiento del ALHT. Tras esta certificación, otras escuelas de la UC se interesaron en obtener la certificación de ALHT. Si bien lograron cumplir con los requisitos, no alcanzaron a formalizar su certificación antes de que entrara en vigor la nueva ley antitabaco, que obliga a todas las escuelas a ser ALHT (9).

\footnotetext{
4 Programa UC Saludable (2001). Resultados encuesta diagnóstico. Estilos de Vida. Alumnos Pontificia Universidad Católica de Chile. [Documento no publicado].
} 
La puesta en marcha de la nueva ley antitabaco durante 2006, se llevó a cabo a través de las siguientes etapas: a) revisión de la literatura para identificar las recomendaciones internacionales; b) establecimiento de metas para promover la nueva ley, y c) planificación de actividades para alcanzar las metas planteadas. El proceso de implementación se desarrolló a lo largo del segundo semestre del año 2006, y contó con la participación del Programa UC Saludable, de los titulares de la Dirección de Asuntos del Personal y la Dirección General Estudiantil, Área Salud, así como de los estudiantes líderes, y del personal administrativo y académico de la UC.

Sobre la base de los antecedentes teóricos obtenidos, se plantearon las siguientes metas: a) lograr el compromiso de los directivos de la UC; b) involucrar a todos los estamentos de la comunidad UC — estudiantes, académicos y administrativos-; c) utilizar diversos medios de comunicación masivos, y d) brindar un espacio de análisis y de reflexión a aquellos directamente afectados por esta normativa: los fumadores.

La planificación de actividades de implementación de la nueva ley chilena antitabaco consideró el trabajo colaborativo y sostenido de diversos miembros clave de la comunidad UC. Para ello, se realizaron reuniones quincenales de coordinación de actividades de implementación. En particular, destaca la realización de una mesa de discusión con la Dirección de Asuntos del Personal y la Vicerrectoría de Asuntos Económicos y Administrativos de la UC, donde se analizó la nueva normativa antitabaco de la UC. Luego, junto con el análisis de la literatura, se discutió la generación de acciones concretas para la implementación de la nueva ley. A partir de la etapa de planificación, se aprobaron las siguientes actividades de implementación de la nueva ley chilena antitabaco: a) participación en el concurso internacional "Déjalo y gana" (Quit $\mathcal{E}$ Win); b) difusión acerca del Día Mundial Sin Tabaco; c) celebración del Foro Debate "Nueva Ley Antitabaco"; d) inclusión de contenidos científicos sobre los efectos del tabaco en la salud, en el sitio en Internet del Programa UC Saludable (www.ucsaludable.cl); e) realización de modificaciones a los espacios públicos de la UC, y f) difusión de la nueva normativa en los medios de comunicación de la UC.

\section{REVISIÓN DE LA LITERATURA. NORMATIVA ANTITABACO EN LA UNIVERSIDAD CATÓLICA}

Se revisó la literatura relativa a experiencias descritas en otras universidades nacionales y/o internacionales en la aplicación de normativas legales antitabaco, utilizando las palabras clave programas/ campañas/normas, tabaco y universidad, en texto completo y en inglés, francés y/o español, con los siguientes resultados en materia de artículos disponibles por base de datos: ocho en Sinal; siete en Cochrane Sistematic Reviews; cinco en Medline, y uno en Proquest Research Library. Se consultaron, asimismo, las bases Alerta al conocimiento y Lilacs, pero no hubo artículos disponibles acerca de este tema.

En términos generales y de acuerdo con lo notificado en la literatura, el ambiente universitario promovería el inicio y aumento del consumo de tabaco, debido a algunos factores como: la visibilidad del tabaco en los campus, la falta de restricciones para su consumo, la presencia de publicidad del tabaco y la venta de productos del tabaco y afines a quienes allí estudian o trabajan. De esta manera, los estudios indican que más de $50 \%$ de los estudiantes aumentan su consumo de tabaco durante el periodo universitario (6). Desde el punto de vista publicitario, el grupo juvenil es particularmente interesante para las empresas tabacaleras, que son muy eficientes para motivar el inicio y la conservación del hábito tabáquico (10). Por otra parte, tanto el personal académico como el administrativo actúan como modelo de consumo de tabaco para sus pares y alumnos, o bien son fumadores pasivos en un entorno que fomenta el cigarrillo.

Las experiencias en otros países indican que la promulgación de regulaciones o de legislación, sin una estrategia de implementación definida, tiene poco efecto en el hábito tabáquico $(7,11)$. Por el contrario, las estrategias en las que se toman medidas múltiples, coordinadas y amplias, reducen efectivamente el consumo de tabaco. Al mismo tiempo, la participación de los directivos de las instituciones y la comunicación directa clara con aquellos afectados por las políticas antitabaco, son estrategias necesarias para contribuir al éxito de la normativa $(7,11)$. La promoción de la salud no surge como un tema interesante y pertinente en la universidad, a menos que se incorpore en las discusiones, decisiones y vida universitaria. El doctor Pedro Pablo Rosso, rector de la UC, ilustró esa idea de la siguiente manera (12):

Los valores no pueden ser transmitidos a los jóvenes mediante declaraciones o disposiciones administrativas, sino con acciones concretas. Por lo mismo, si queremos que los estudiantes opten por estilos de vida saludables, las instituciones de educación superior deben ser capaces de motivarlos y orientarlos a partir de ofertas atractivas $y$, al mismo tiempo, coherentes con la cultura institucional. Es decir, sólo una universidad saludable puede 
educar a sus estudiantes en conductas saludables y para eso las conductas que propone deben ser parte de la cultura institucional.

Diversas recomendaciones generales se han desarrollado para el control del consumo de tabaco en las organizaciones (cuadro 1). La mayoría están orientadas a impactar en la población y a ser evaluadas en forma global, más que individual $(7,11$, 13). En cuanto al uso de medios de comunicación masivos para el control de tabaco, existe cierta evidencia de que éstos pueden ser eficaces para prevenir el hábito de fumar en personas jóvenes, aunque la evidencia global no es sólida $(14,15)$.

Para el contexto universitario, existen ocho recomendaciones específicas de control de venta y consumo de tabaco, descritas por las organizaciones estadounidenses American Center Society (ACS), American College Health Association (ACHA) y National Center on Addiction and Substance Abuse (CASA), que prohíben su consumo: a) al interior de los edificios del campus; b) al interior de las residencias de estudiantes, y c) en puertas de acceso a edificios del campus. Asimismo prohíben: a) la venta de tabaco; b) la publicidad en torno al tabaco en edificios y en cualquier medio de comunicación interna de la universidad, y c) la publicidad del tabaco y auspicio de eventos por parte de empresas tabacaleras. Por otra parte, exigen la reducción de productos disponibles de compañías tabacaleras, y el pago del tratamiento para dejar de fumar a jóvenes, como parte de su seguro de salud estudiantil. Estas recomendaciones, según lo notificado por un estudio longitudinal realizado en 50 universidades públicas de diferentes entidades de los Estados Unidos de América, han demostrado ser eficaces en el control del consumo de tabaco al interior del ámbito universitario (6).

Finalmente, diversos autores señalan que una campaña de control de tabaco exitosa requiere de una sólida base teórica, que la guíe y sustente (1316). Con el tiempo, han surgido diferentes enfoques teóricos que han pasado de la perspectiva puramente cognitiva, a miradas más integrales y multidisciplinarias. El primero corresponde al enfoque racional, que se limita a entregar información a la comunidad sobre los efectos nocivos del consumo de tabaco.

Posteriormente apareció el enfoque de aprendizaje social, que procede de la teoría del mismo nombre de Bandura y es, en la actualidad, uno de los principales modelos que sustentan las estrategias de prevención de consumo de tabaco. Aquí destaca la relevancia del grupo de pares y el proceso de socialización para el inicio, conservación y cambio de conducta. De esta manera, se subraya la entrega de opciones de conductas alternativas a la de fumar y
CUADRO 1. Recomendaciones generales para el control del consumo de tabaco en organizaciones, Estados Unidos, 2003

a. Colocación de avisos informando acerca de la política y carteles con la indicación de no fumar.

b. Eliminación de ceniceros.

c. Avisos acerca de los efectos de fumar y de no fumar sobre la salud.

d. Campañas educativas para empleados con información de nueva normativa y asesoría en promoción de la salud.

e. Asesoría con expertos para dejar de fumar.

f. Áreas específicas para consumo claramente especificadas y con ventilación separada.

g. Restricciones para la compra de tabaco.

h. Difusión en medios de comunicación masiva sobre la normativa y los efectos perjudiciales de fumar.

i. Avisos y solicitudes amables a los fumadores para que dejen el hábito, de parte de administradores y/o no fumadores.

Fuente: Serra C, Cabezas C, Bonfill X, Pladevall-Vila M. Intervenciones para prevenir el hábito de fumar en lugares públicos (Revisión Cochrane traducida de The Cochrane Library, 2007 Issue 4. Chichester, UK: John Wiley \& Sons, Ltd.). Sowden A, Stead D. Community interventions for preventing smoking in young people (Cochrane Review). En: The Cochrane Review Library, Issue 4, 2003. Oxford: Update Software. Thomas R, Perera R. School-based programmes for preventing smoking. Cochrane Database of Systematic Reviews 2006, Issue 3. Art. No.: CD001293. DOI: 10.1002/14651858.CD001293.pub2.

la presentación de modelos de rol positivos donde se rechaza el consumo en situaciones típicas.

Existe, además, el enfoque afectivo, que fortalece la autoestima y la autovaloración de las personas, para luego incidir en el mejoramiento de su autoeficacia. Aquí se plantea que la persona desea rechazar el consumo de tabaco y efectivamente lograrlo, tras el desarrollo de capacidades para la toma de decisiones y de habilidades interpersonales. Un último enfoque incorporado es el de la comercialización social, que resulta interesante e innovador puesto que aplica principios de publicidad y mercado al consumo de tabaco. Propone la "venta" de conductas sanitarias positivas - como rechazar el consumo de tabaco entre amigos-, entendiéndose éstas como un concepto atractivo para el mercado y capaz de competir con la publicidad existente que incita hacia el consumo de tabaco $(17,18)$.

\section{LOGROS DEL PROGRAMA UC SALUDABLE EN LA IMPLEMENTACIÓN DE LA LEY ANTITABACO EN LA UNIVERSIDAD CATÓLICA}

A continuación se describen las actividades desarrolladas por el Programa UC Saludable, a partir del proceso de implementación de la nueva ley chilena antitabaco: 


\section{Concurso internacional Déjalo y gana}

Este concurso Déjalo y gana (del inglés Quit \& Win) corresponde a una estrategia para dejar de fumar auspiciada desde 1994 por la Organización Mundial de la Salud y coordinada mundialmente por el Instituto de Salud Pública de Finlandia. Consiste en el libre compromiso de los participantes mayores de 18 años de no fumar por 30 días. Cuenta con premios internacionales, regionales y nacionales, estos últimos bajo la coordinación del Ministerio de Salud de Chile.

Desde 1998 la UC ha colaborado en este concurso. Además, a partir del año 2000 desarrolló en forma simultánea su propio concurso interno, que se suma a las premiaciones recién mencionadas (10) y que durante 2006 tuvo especial realce por ser el año en que entró en vigor la nueva ley antitabaco. El Programa UC Saludable encabezó este esfuerzo en el ámbito universitario, logrando la participación de 128 personas de la comunidad UC-entre académicos, estudiantes y administrativos. Se premió a tres concursantes escogidos al azar, por haber cumplido con los 30 días de abstinencia confirmados mediante un examen de orina específico. Se estima que ninguna de las personas inscritas consumió tabaco durante los 30 días del concurso, pero no se llevó a cabo una evaluación ni el seguimiento de estas personas.
Por otra parte, la UC fue centro de difusión e inscripción para todas las instancias de premiación del concurso: interno, nacional, latinoamericano e internacional. En el cuadro 2 se muestran los diversos estamentos y organizaciones universitarias que colaboraron en la realización del concurso.

\section{Día Mundial Sin Tabaco}

El 31 de mayo se celebra el Día Mundial Sin Tabaco; con la mira de aprovechar esta fecha, se desarrolló una campaña antitabaco mediante la entrega de 2000 volantes a los automovilistas que ingresaron ese día a todos los campus de la UC, entre las ocho y las nueve de la mañana. De esta manera, en forma anticipada a la entrada en vigor de la nueva ley, se instaló progresivamente el tema como asunto interesante y necesario de abordar.

\section{Foro Debate “Nueva Ley Antitabaco"}

Con motivo de la premiación del concurso interno universitario Déjalo y gana, se organizó un foro debate abierto a toda la comunidad UC, en torno a la puesta en marcha de la nueva ley antitabaco. Para la ocasión, se invitó al asesor del ámbito salud de la Cámara de Diputados, al médico jefe del

CUADRO 2. Colaboradores por estrategia de implementación de la nueva ley antitabaco. Pontificia Universidad Católica de Chile, Santiago, 2006

\begin{tabular}{ll}
\hline \multicolumn{1}{c}{ Estrategia } & \multicolumn{1}{c}{ Colaboradores } \\
\hline Concurso internacional "Déjalo y gana" & Ministerio de Salud \\
& Pontificia Universidad Católica de Chile (UC) \\
& Departamento de Salud Pública \\
& Programa UC Saludable \\
& Federación de Estudiantes (FEUC) \\
& Centro de alumnos Medicina (CEMUC) \\
& Centro de alumnos Enfermería (CAEO) \\
& Dirección Asuntos del Personal \\
Día Mundial Sin Tabaco & Dirección General Estudiantil Salud (DGE Salud) \\
& Programa UC Saludable \\
Foro Debate "Nueva ley antitabaco" & Dirección de Administración de Campus UC \\
& Programa UC Saludable \\
& Federación de Estudiantes UC (FEUC) \\
Artículos sobre consumo de tabaco & Dirección General Estudiantil Salud (DGE Salud) \\
Incorporación de avisos públicos en la UC & Departamento de Salud Pública UC \\
de nueva ley antitabaco & Dirección Asuntos del Personal UC \\
& Sitio en Internet del Programa UC Saludable \\
& Pontificia Universidad Católica de Chile (UC) \\
& Programa UC Saludable \\
& Dirección Asuntos del Personal \\
\hline
\end{tabular}


Departamento de Salud Pública de la UC y a la titular de la Dirección General Estudiantil, Área Salud, de la UC. A este foro debate asistieron todos los estamentos de la UC y sus principales logros fueron: a) instalar en forma concreta el tema de la nueva ley; b) permitir la participación simultánea de estudiantes, administrativos y académicos; c) contribuir al intercambio de opinión experta para el control de consumo del tabaco, y d) permitir la expresión de inquietudes respecto de la nueva ley, fundamentalmente por parte del grupo estudiantil. Dichos cuestionamientos y sugerencias resultaron directrices para el trabajo posterior de implementación de la normativa interna antitabaco. En el cuadro 2 se muestran los diversos estamentos y organizaciones universitarias que colaboraron en la realización del concurso.

\section{Inclusión de artículos sobre el consumo de tabaco en Internet}

El Programa UC Saludable cuenta desde su origen con el sitio en Internet www.ucsaludable.cl, con un promedio de 1018 visitas mensuales durante 2006 y 2007; por su parte, la UC posee extensas salas de computadores con acceso a Internet de libre uso para estudiantes, y los académicos y administrativos también disponen de acceso; de otro lado, existe conexión con red e inalámbrica en todos los campus de la UC.

En el sitio en Internet, en la liga correspondiente a "tabaco y otras drogas", de enero a diciembre de 2006 se incluyeron mensualmente artículos científicos referidos a la relevancia de no iniciar, o bien de reducir o suspender el consumo de tabaco y de los efectos beneficiosos asociados a dichos procesos, considerando también a quienes fuman pasivamente.

\section{Modificaciones a espacios públicos de la UC}

En la mesa de discusión con la Dirección de Asuntos del Personal de la UC, se determinaron modificaciones para los espacios públicos como la eliminación de ceniceros en espacios cerrados, y la prohibición de exhibir publicidad en torno al tabaco en la UC y de vender de tabaco a menores de 18 años. Al mismo tiempo, se determinó que la gerencia administrativa se encargaría de fiscalizar el cumplimiento de la ley.

Para incorporar el concepto de promoción de la salud en avisos públicos antitabaco, el Programa UC Saludable diseñó contenidos breves sobre los beneficios de dejar de fumar y el daño producido en fumadores pasivos. Se determinó que estos conteni- dos serían elaborados como avisos públicos y que se sumarían a los tradicionales carteles de "No fumar" que existían en la UC. Algunos ejemplos del contenido de estos mensajes, fueron los siguientes:

- Mira alrededor, este sector no es para fumar, protege y respeta a quienes te rodean.

- UC Saludable te recuerda: todos los edificios son ambientes libres de humo de tabaco, excepto oficinas individuales con ventilación.

- UC Saludable te recuerda: mantengamos este sector libre del humo de tabaco.

- Un ambiente saludable en la Universidad es esfuerzo de todos; respeta y apoya el cumplimiento de la ley antitabaco.

\section{Difusión de la nueva normativa en los medios de comunicación de la UC}

Todas las actividades desarrolladas se dieron a conocer a través de diversos medios de comunicación internos de la UC. Se utilizaron las secciones "agenda" y "noticias" del Portal de la Universidad Católica (www.uc.cl), así como los sitios en Internet de la Escuela de Enfermería, la Federación de Estudiantes (FEUC), el Centro de Alumnos de Medicina (CEMUC) y el Centro de Alumnos de Enfermería (CAEO), de la UC. Asimismo, se aprovecharon el Boletín de la Dirección General Estudiantil (DGE) Salud y los recibos de pago de sueldos para administrativos y académicos. También se hizo entrega de una carta certificada a los directivos, académicos, administrativos y estudiantes, en la cual se adjuntaba la nueva normativa antitabaco UC.

\section{CONCLUSIONES}

La incorporación de la perspectiva de promoción de la salud aportó una mirada complementaria e innovadora para la implementación de la nueva ley chilena antitabaco al interior de la UC. La metodología utilizada permitió el trabajo con todos los estamentos de la UC; en particular, los estudiantes lograron expresar sus aprehensiones, inquietudes y sugerencias en torno a la implementación de la nueva ley. La revisión de la literatura brindó un marco conceptual al trabajo, en especial para el análisis de la nueva ley y de la normativa interna. Por su parte, la mesa de discusión permitió resolver inquietudes, contrastar opiniones y acordar estrategias de acción. Se utilizaron, además, estrategias y medios de comunicación diversos para la difusión de las actividades y de la normativa antitabaco.

De esta experiencia surgen algunas consideraciones que pueden ser útiles para otros casos de im- 
plementación de una normativa antitabaco en ámbitos universitarios. En primer lugar, es necesario utilizar estrategias que idealmente hayan sido probadas como eficaces en la literatura. Enseguida, se debe responder a constructos teóricos sólidos respecto de la adquisición y conservación del consumo de tabaco en adolescentes y adultos. En tercer lugar, los programas deben ser suficientemente flexibles para enfrentar diferentes escenarios y comunidades. Al mismo tiempo, las estrategias seleccionadas deben adaptarse a las necesidades de cada grupo en particular. Por último, se requiere de mayor rigurosidad metodológica que permita evaluaciones de resultado y de proceso reales respecto de las estrategias más efectivas a utilizar.

Finalmente, se asumen desafíos y tareas pendientes para garantizar que este trabajo perdure y resulte efectivo, entre los que destacan la permanencia de las estrategias comunicacionales a lo largo del tiempo, la consideración de estrategias específicas para subgrupos de la UC y el mejoramiento del trabajo multidisciplinario desarrollado. Es importante conservar e incrementar el compromiso de los equipos directivos en la incorporación permanente de la promoción de la salud a sus actividades y cultura organizacional. Es necesario evaluar periódicamente la prevalencia del consumo de tabaco en la comunidad universitaria.

Existen dos debilidades en la implementación de la nueva ley antitabaco: una, es que se debe reformular la posibilidad de fumar tabaco en áreas específicas dentro de la UC. Esto se contradice con las recomendaciones internacionales y está permitido según la nueva ley chilena antitabaco (la Universidad no es un espacio totalmente libre de humo de tabaco). La otra, es que se deben plantear estrategias concretas de apoyo para que fumadores interesados logren dejar el hábito. Hasta ahora el apoyo se reduce a la orientación y derivación a centros especializados de adicción a la nicotina, lo cual sin lugar a dudas es insuficiente para las posibles necesidades de la población fumadora de la UC.

Por último, se reconoce que la evaluación pendiente de la implementación de la nueva normativa antitabaco en la UC constituye un gran reto.
Para ello, habrá que considerar la normativa interna UC, las recomendaciones internacionales resultantes de la revisión de la literatura, las modificaciones a los espacios comunes acordadas con directivos y la supervisión del cumplimiento de la normativa por parte de miembros de la UC. Además, debería evaluarse la costo-efectividad de estas intervenciones.

Sólo así, tanto la nueva ley antitabaco como cualquier nueva empresa de promoción de la salud, podrán alcanzar el éxito y, como meta última, contribuir al mejoramiento de la salud y calidad de vida de la comunidad.

\section{SYNOPSIS}

\section{How to implement Chile's new anti-tobacco law within the university}

On the 16th of May 2006, the Diario Oficial de la República de Chile (Official News of the Republic of Chile), published Law No. 20.105, which modified Law No. 19.419 regarding advertising and consumption of tobacco. Since it entered into force, different institutions and public spaces have had to enact its regulations. The purpose of this article is to share how the new anti-tobacco law is being implemented, with a health promotion angle, by the Pontificia Universidad Católica de Chile (UC). As such, we highlight key findings from a review of the literature on the subject and describe the implementation strategies developed and substantiated in theory as well as by how they address the concerns of the university community. The main accomplishments of the anti-tobacco implementation were: reaching a compromise between the university directorate and the student leaders, who in turn motivated the student body to participate during the large-scale roll-out of the new law; the participation of all of the UC stakeholders; utilizing the varied methods of mass communication; and lastly, providing an opportunity for reflection and analysis for those directly affected: the smokers. The challenge is to evaluate the strategies implemented in the incorporation of the new Chilean anti-tobacco law.

Key words: student health services, health promotion, tobacco control campaigns, Chile.

\section{REFERENCIAS}

1. Diario Oficial de la República de Chile. Ley $\mathrm{N}^{\circ}$ 20.105. Materias relativas a la publicidad y el consumo del tabaco. Subsecretaría de Salud Pública. Ministerio de Salud. República de Chile. [Sitio en Internet] Hallado en: http://www. diariooficial.cl/ Acceso el 7 julio 2006.
2. Vio F, Lange I. Guía para universidades promotoras de la salud y otras instituciones de educación superior. $1^{\mathrm{a}}$ ed. Santiago: Ministerio de Salud; 2006.

3. Organización Panamericana de la Salud. Promoción de la salud, un enfoque innovador y eficaz. Noticias e información del centenario, información de prensa. 2002. [Sitio en Internet] Hallado en: http:/ / www.paho.org/Spanish/DPI/ 100/100feature47.htm. Acceso el 23 septiembre 2006.

4. Ottawa Charter for Health Promotion. Can J of Pub Health. 1986;77(6):425-30. 
5. Organización Panamericana de la Salud. Organización Mundial de la Salud. [publicación periódica en línea] Consejo Directivo No.46. Sesión del Comité Regional No.57. Hallado en: URL: http:// www.paho.org

6. Halperin A, Rigotti N. US public universities compliance with recommended tobacco-control policies. J of Am Col Health. 2003;51(5):181-88.

7. Serra C, Cabezas C, Bonfill X, PladevallVila M. Intervenciones para prevenir el hábito de fumar en lugares públicos (Revisión Cochrane traducida de The Cochrane Library, 2007 Issue 4. Chichester, UK: John Wiley \& Sons, Ltd.). En: La Biblioteca Cochrane Plus, número 4, 2007. Oxford, Update Software Ltd. Hallado en: http://www.update-software.com. Acceso el 24 agosto 2006.

8. Diario Oficial de la República de Chile. Ley $\mathrm{N}^{\circ}$ 19.419. Materias relativas al consumo del tabaco. Subsecretaría de Salud Pública. Ministerio de Salud. República de Chile. [Sitio en Internet] Hallado en: http://www.diariooficial.cl/ Acceso el 7 julio 2006.

9. Lange I, Alsina N, Contreras A, Vial B, Muñoz M, Urrutia MT. UC Saludable: vive tu equilibrio. Rev Horizonte de Enf. 2002;13:71-6.

10. Lovato C, Linn G, Stead LF, Best A. Impact of tobacco advertising and promo- tion on increasing adolescent smoking behaviours. Cochrane Database of Systematic Reviews 2003, Issue 4. Art. No.: CD003439. DOI: 10.1002/14651858. CD003439. Hallado en: http://www. cochrane.org/reviews/en/ab003439. html. Acceso el 15 agosto 2006.

11. Sowden A, Stead D. Community interventions for preventing smoking in young people (Cochrane Review). En: The Cochrane Review Library, Issue 4 2003. Oxford: Update Software. Hallado en: http://sciencedirect.com/ Acceso el 16 agosto 2006.

12. Rosso P. Discurso por Lanzamiento Oficial Guía para Universidades Promotoras de la Salud. Rectoría. Pontificia Universidad Católica de Chile. Santiago, Chile. [Sitio en Internet] Hallado en: URL: http://www.ucsaludable.cl. Acceso el 21 septiembre 2006.

13. Thomas R, Perera R. School-based programmes for preventing smoking. Cochrane Database of Systematic Reviews 2006, Issue 3. Art. No.: CD001293. DOI: 10.1002/14651858.CD001293.pub2. Hallado en: http://mrw.interscience. wiley.com/cochrane/clsysrev/articles/ CD001293/frame.html. Acceso el 15 agosto 2006

14. Sowden AJ, Arblaster L. Intervenciones en medios de comunicación masivos para prevenir el hábito de fumar en per- sonas jóvenes (Revisión Cochrane traducida de The Cochrane Library, 2007 Issue 4. Chichester, UK: John Wiley \& Sons, Ltd.). En: La Biblioteca Cochrane Plus, número 4, 2007. Oxford, Update Software Ltd. Disponible en: http:// www.update-software.com. Acceso el 16 agosto 2006.

15. Tyas SL, Pederson LL. Factores psicológicos en la iniciación de fumar en adolescentes: revisión crítica y análisis de literatura. Toronto: The Ontario Tobacco Research Unit; 1997.

16. Atkin C, Wallack L, eds. Medios de comunicación masiva y salud pública. Complejidades y conflictos. London: Sage; 1990.

17. Straub D, Hills N, Thompson P, Moscicki $\mathrm{AB}$. Effects of pro and anti-tobacco advertising on nonsmoking adolescents intentions to smoke. Journal of Adolescent Health. 2003; 32:36-43.

18. Weiss JW, Cen S, Schuster D, Unger J, Johnson A, Mouttapa M, et al. Longitudinal effects of pro-tobacco and antitobacco messages on adolescent smoking susceptibility. Nicotine \& Tobacco Research. 2006; 8(3):455-65. 